\title{
Perfil microbiológico dos pacientes submetidos à cultura de vigilância ativa em um hospital universitário \\ da Região Sudeste de Minas Gerais
}

\section{Hיthevista}

Microbiological profile of patients undergoing active surveillance culture in a university hospital in the Southeastern Region of Minas Gerais

${ }^{1}$ Enfermeira do Setor de Vigilância em Saúde e Segurança do Paciente do Hospital Universitário da Universidade Federal de Juiz de Fora, Brasil. Especialista em Vigilância Epidemiológica pela Escola Superior de Estatística da Bahia, e em Controle de Infecção e Gestão em Saúde pelo Instituto Nacional de Ensino e Pesquisa.

${ }^{2}$ Enfermeira do Setor de Vigilância em Saúde e Segurança do Paciente do Hospital Universitário da Universidade Federal de Juiz de Fora, Brasil. Especialista em enfermagem em UTI, pela Unyleya.

${ }^{3}$ Enfermeira do Setor de Vigilância em Saúde e Segurança do Paciente do Hospital Universitário da Universidade Federal de Juiz de Fora, Brasil. Doutora em Saúde pela Universidade Federal de Juiz de Fora, Brasil.

\section{RESUMO}

Objetivo: Identificar o perfil microbiológico dos pacientes submetidos à cultura de vigilância ativa. Material e métodos: Estudo de prevalência realizado em um hospital universitário da Região Sudeste de Minas Gerais no período de março a dezembro de 2018, com os pacientes elegíveis pelos critérios pré-estabelecidos pelo Serviço de Controle de Infecção Hospitalar da referida unidade, submetidos à cultura de vigilância por meio de swab retal e nasal. Os critérios definidos foram: pacientes transferidos de outra instituição com permanência maior que 96 horas; pacientes transferidos de outra instituição com internação mínima de 48 horas e submetidos a algum dispositivo invasivo; realização de terapia renal substitutiva; passagem por Unidade de Terapia Intensiva (nos últimos 90 dias com permanência mínima de 72h;internação prévia nos últimos 90 dias com permanência mínima de 30 dias. 0 banco de dados foi estruturado e analisado por meio do Excel e as análises estatísticas pelo MedCalc. Resultados: Foram identificados 591 pacientes que atendiam aos critérios para a realização da cultura de vigilância, destes 25,4\% foram positivos. Os critérios mais frequentes para realização de cultura foram: pacientes transferidos de outra instituição com permanência maior que 96 horas e pacientes com passagem por unidade de terapia intensiva nos últimos 90 dias com permanência mínima de $72 \mathrm{~h}$. Os Principais microrganismos identificados foram: Staphylococcus aureus resistente à meticilina, Klebsiella pneumoniae e Acinetobacter baumannii resistentes à carbapenêmicos com 38,3\%, $31,2 \%$ e $25,3 \%$, respectivamente. Conclusão: A cultura de vigilância ativa contribuiu para a detecção precoce de microrganismos resistentes, permitindo a prevenção precoce, favorecendo a redução da disseminação cruzada. 0 banco de dados com resultados das culturas de vigilância é uma estratégia importante, pois caso ocorra reinternações desses pacientes, as equipes de controle de infecção e assistencial podem identificar aqueles já infectados/colonizados e instaurar as medidas de controle na admissão dos mesmos.

Palavras-chave: vigilância, controle de infecção, infecção hospitalar

\section{ABSTRACT}

Objective: To identify the microbiological profile of patients undergone active surveillance culture. Material and Methods: Prevalence study was performed at University Hospital in the Southeastern Region of Minas Gerais from March to December 2018, with eligible patients according to criteria established by the Hospital Infection Control Service of this unit, submitted to culture of surveillance by rectal and nasal swabs. The criteria were defined as: patients transferred from another institution with permanence higher than 96 hours; patients transferred from another institution at least 48 hours of hospitalization and submitted to some invasive device; replacement renal therapy; staying intensive care unit at least 72 hours by last 90 days; previously admitted by last 90 days at least of 30 days of hospitalization. The database was structured and analyzed through Excel and statistical analyzes by Med Calc. Results: 591 patients were identified and presented criteria for performing the surveillance culture, being $25.4 \%$ as positive. Most of frequent criteria for performing culture were: patients transferred from another institution with permanence higher than 96 hours and staying intensive care unit at least 72 hours by last 90 days. The microorganisms were mainly identified: methicillin resistant Staphylococcus aureus, Klebsiella pneumoniae and Acinetobacter baumannii resistant to carbapenems with $38.3 \%, 31.2 \%$ and $25.3 \%$, respectively. Conclusion: The culture of active surveillance contributed to the early detection of resistant microorganisms, allowing early prevention, favoring the reduction of cross-dissemination. The database with results of surveillance cultures is an important strategy, because if rehospitalizations of these patients occur, infection control and care teams will be able to identify patients have already infected or colonized and make control behaviors on their admission.

Keywords: surveillance, infection control, cross infection 


\section{INTRODUÇÃO}

As Infecções Relacionadas à Assistência à Saúde (IRAS) são infecções adquiridas após a admissão do paciente em âmbito hospitalar e que têm seu aparecimento durante a internação ou após a alta e estão associadas também a ambientes extra hospitalares, onde são realizados procedimentos de cuidados à saúde, como clínicas médicas, home care, entre outros (BRASIL, 1998). Segundo a Portaria 2.616/98, caso o período de incubação da infecção seja desconhecido ou não havendo evidência de infecção na admissão, as infecções subsequentes que se apresentarem a partir de 72 horas da internação serão consideradas Infecções Hospitalares (BRASIL, 1998).

A emergência de microrganismos resistentes a diversas classes de antimicrobianos chamados de multirresistentes reflete em um dos mais graves desafios que atingem os hospitais brasileiros, principalmente os universitários (NOGUEIRA et al., 2009). Estes microrganismos multirresistentes são introduzidos nas instituições de saúde de duas formas principais: devido à colonização e/ou infecção de pacientes por microrganismos multirresistentes e devido à pressão seletiva gerada pelos agentes antimicrobianos (ANVISA, 2007).

De acordo com a Agência Nacional de Vigilância Sanitária (ANVISA, 2007), as IRAS representam um dos mais importantes problemas da saúde pública, pois são os eventos adversos associados à assistência à saúde mais frequentes. Segundo Lachhab et al. (2017), a mortalidade pode ser atribuível a infecções por patógenos multirresistentes. Nos Estados Unidos e Europa a estimativa do número de mortes devido à resistência microbiana foi estimada em 50.000 mortes por ano e de 700.000 no mundo (REVIEW ON ANTIMICROBIAL RESISTANCE, 2014). O crescimento da mortalidade e o aumento do tempo de internação do paciente, geralmente, vêm associados com alguma IRAS e repercutem diretamente nos custos diretos e indiretos associados à assistência à saúde, constituindo dessa forma um fardo social e econômico significativo tanto para os pacientes quanto para o sistema de saúde (FIGUEIREDO, 2012).

Conhecero perfil desusceptibilidade aos antimicrobianos é outro aspecto essencial para que o profissional de saúde possa realizar ações de prevenção e controle de IRAS . Tal ato possibilita o uso racional da terapia antimicrobiana na instituição, pois ao conhecer o perfil de resistência do seu local de trabalho, a prescrição e a dispensação de antibióticos se basearão nos microrganismos encontrados de forma mais frequente, assim como a resistência dos mesmos. Os programas de vigilância que, entre outras medidas, avaliam o perfil de susceptibilidade dos microrganismos são fundamentais, visto que a disseminação de bactérias multirresistentes alcançam cada vez mais as instituições hospitalares (PAIM; LORENZINI, 2014).

Segundo Martins et al. (2014), a vigilância é muito relevante na prevenção e controle da transmissão cruzada entre os microrganismos multirresistentes, possibilitando a identificação de patógenos emergentes, o monitoramento das tendências epidemiológicas e avaliar a eficácia das intervenções. Álvarez-Lerma et al. (2015) ressaltam que a identificação de microrganismos multirresistentes encontrados nas amostras clínicas é pouco eficaz, detectando entre 10 a $30 \%$ os pacientes colonizados por tais agentes. Colonizado é o indivíduo que é portador do microrganismo mas que não desenvolve a doença infecciosa e pode representar um agente de disseminação do mesmo (ANVISA, 2007). Já a vigilância ativa caracteriza-se como uma estratégia para conhecer, detectar e implementar medidas quando do surgimento de eventos adversos infecciosos relacionados ao paciente. É uma estratégia recomendada pela Anvisa que seja implementada pelo Serviço de Controle de Infecção Hospitalar (SCIH) das instituições de saúde.

O protocolo de cultura de vigilância ativa consiste na coleta de amostras de pacientes internados ou que necessitam de internação, principalmente em unidades de terapia intensiva (UTI) para identificar a colonização destes por patógenos em sítios não estéreis e monitorar o aparecimento de microrganismos multirresistentes após o período de permanência no âmbito hospitalar (ANVISA, 2007).

Estudos apontam que a cultura de vigilância ativa é a abordagem com maior nível de sensibilidade para detectar pacientes colonizados. É recomendado que ela seja realizada em todos os pacientes admitidos no hospital (FURUNO et al., 2006). No entanto, o tempo gasto com a execução dos testes microbiológicos, assim como seu alto custo, dificultam a implementação desse método na maior parte das instituições hospitalares. Assim, a utilização dos critérios clínicos segue sendo a opção mais viável e de menor custo, comparada com a vigilância ativa (MORGAN et al., 2010).

Martins et al. (2014) afirmam que, de acordo com o perfil epidemiológico da instituição, a cultura de vigilância ativa é uma estratégia capaz de prevenir e controlar microrganismos multirresistentes. Porém deve contar com o apoio da direção clínica e administrativa, recursos humanos capacitados para a coleta, um laboratório de microbiologia capaz de analisar e processar as culturas, comunicação ágil e eficaz entre o SCIH e o laboratório, a instituição das medidas de precaução de contato e sua correta utilização e, por fim, a auditoria para verificação da adesão a essas medidas.

O SCIH do hospital deverá analisar o perfil epidemiológico dos seus pacientes e identificar qual será a população-alvo para a realização da cultura de vigilância. Fatores de risco, como internação prévia em outras instituições ou nas unidades de terapia intensiva, uso prolongado de terapia antimicrobiana e uso de dispositivos invasivos devem ser levados em conta, pois são capazes de contaminar pacientes e alterar o perfil de resistência dos já colonizados. Os métodos para obtenção das culturas 
variam dependendo do microrganismo multirresistente de interesse.

O objetivo deste estudo foi identificar o perfil microbiológico dos pacientes internados submetidos à cultura de vigilância ativa no HU.

\section{MATERIAL E MÉTODOS}

Trata-se de um estudo retrospectivo, desenvolvido pelos técnicos do Serviço de Controle de Infecção Hospitalar (SCIH) do Hospital Universitário da Universidade Federal de Juiz de Fora - MG (HU-UFJF). O HU-UFJF disponibiliza, atualmente, uma capacidade instalada e ocupacional de 140 leitos, sendo 09 (nove) destes em Unidade de Terapia Intensiva e 05 (cinco) na Unidade de Transplante de Medula Óssea. Vale ressaltar que o HU-UFJF é referência na região para as doenças infecto- parasitárias, perfazendo uma média de 500 internações/mês.

Em 2016, O SCIH, do hospital em estudo, elaborou um protocolo para admissão de pacientes procedentes de internação em outros hospitais, objetivando minimizar a disseminação de microrganismos multirresistentes.

Os critérios para realizar a coleta da cultura de vigilância definidos pelo SCIH foram:

Pacientes transferidos de outra instituição com permanência maior que 96 horas;

Pacientes transferidos de outra instituição com internação mínima de 48 horas e submetidos a algum dispositivo invasivo;

Realização de terapia renal substitutiva;

Passagem por unidade de terapia intensiva (UTI) nos últimos 90 dias com permanência mínima de $72 \mathrm{~h}$; e

Internação prévia nos últimos 90 dias com permanência mínima de 30 dias.

De acordo com o protocolo do SCIH da instituição, definiu-se quais os microrganismos seriam pesquisados na cultura de Vigilância. Foram realizadas culturas em meios seletivos buscando a identificação de MRSA (Staphylococcus aureus resistente à meticilina) no swab nasal e Bactérias Gram-Negativas (BGNs) resistentes à carbapenêmicos e VRE (Enterococcus resistente à Vancomicina) no swab anal. O meio de cultura utilizado no laboratório da instituição para identificação dos microrganismos foi Caldo Soja Tripticaseína (TSB) + Ertapenem, Ágar Columbia Ácido Nalidíxico (CNA) e Ágar Sal Manitol.

A coleta da cultura de vigilância foi realizada através de dois swabs: nasal e anal. A escolha destes dois sítios partiu das indicações que a literatura nos traz em relação aos sítios não estéreis em que há possibilidade de ter MRSA, VRE e BGN resistente à carbapenêmicos (CURSINO et al., 2012; DALBEN et al., 2010).

A partir da identificação na admissão do paciente de algum critério para realização da coleta da cultura de vigilância, a Enfermeira da unidade de internação registra esta informação no sistema da instituição que serve de comunicação com o SCIH e já institui as precauções de contato. Após a coleta dos swabs realizada pela Enfermeira da unidade, o material é enviado para o laboratório e o resultado é liberado em média com cinco dias. O laboratório comunica ao $\mathrm{SCIH}$ caso resulte em alguma bactéria gram negativa resistente à carbapenêmicos, MRSA, VRE e/ou Stenotrophomonas maltophilia.

Os resultados informados pelo laboratório alimentam, diariamente, uma planilha com as seguintes informações: Nome do paciente, número do prontuário, sexo, idade, critério utilizado, datas da solicitação, coleta e resultado da cultura de vigilância.

O banco de dados foi estruturado e analisado por meio do Excel e as análises estatísticas pelo MedCalc.

Trata-se de análise realizada pelo grupo de técnicos do SCIH do HU-UFJF de setor. A proposta foi submetida ao Comitê de Ética do HU-UFJF.

\section{RESULTADOS}

No período de março a dezembro de 2018 foram realizadas 591 culturas de vigilância o que equivale a $15 \%$ dos pacientes admitidos neste período no hospital. Os critérios mais solicitados foram: 1-Transferidos de outra instituição com permanência maior que 96 horas e 4-Passagem por UTI nos últimos 90 dias com permanência mínima de $72 \mathrm{~h}$, com $65,6 \%$ e $39,6 \%$, respectivamente. Dos pacientes eleitos pelo critério 1 , $20,9 \%$ apresentaram cultura positiva e os do critério 4, 39,6\%. Amostras de pacientes cujo critério não foi informado representaram $13 \%$ das amostras, sendo 23,4\% positivas (Tabela 1 ).

As linhagens mais frequentes isoladas nas amostras dos pacientes que apresentaram o critério 1 foram: Staphylococcus aureus resistente à Meticilina (MRSA), Acinetobacter baumannii, Klebsiella pneumoniae e Enterococcus resistente à Vancomicina (VRE) com $46,9 \%, 22,2 \%, 21,0$ e 17,3\%, respectivamente. Entre os pacientes que apresentaram o critério 4 , os principais foram: Klebsiella pneumoniae (50\%), Acinetobacter baumannii $(28,3 \%)$ VRE $(23,9)$. Nos demais critérios, pode-se destacar MRSA, com 61,5\% (Tabela 2).

As solicitações de cultura foram mais frequentes entre os pacientes na faixa etária acima de 40 anos (Tabela 3).

A idade média dos pacientes com resultado de cultura positiva foi 57,9 anos. Houve diferença estatisticamente significativa entre idade e resultado da cultura dos pacientes com que apresentaram o critério 1 (Tabela 4 ).

Nos demais critérios não houve diferença significativa. Quanto ao sexo, não houve diferença significativa nos resultados das culturas em nenhum dos critérios solicitados (Tabela 5). 
Tabela 1: Critérios para realização de cultura de vigilância e resultados

\begin{tabular}{|c|c|c|c|c|c|c|}
\hline \multirow[t]{2}{*}{ Critérios para cultura } & \multicolumn{2}{|c|}{$(+)$} & \multicolumn{2}{|c|}{$(-)$} & \multicolumn{2}{|c|}{ Total } \\
\hline & $n^{\circ}$ & $\%$ & $n^{\circ}$ & $\%$ & $\mathrm{n}^{\circ}$ & $\%$ \\
\hline (1) & 81 & 20,9 & 307 & 79,1 & 388 & 100,0 \\
\hline$(2)$ & 4 & 44,4 & 5 & 55,6 & 9 & 100,0 \\
\hline (3) & 2 & 40,0 & 3 & 60,0 & 5 & 100,0 \\
\hline (4) & 42 & 39,6 & 64 & 60,4 & 106 & 100,0 \\
\hline$(5)$ & 3 & 50,0 & 3 & 50,0 & 6 & 100,0 \\
\hline Sem informação sobre o critério & 18 & 23,4 & 59 & 59 & 77 & 100,0 \\
\hline Total & 150 & 25,4 & 441 & 74,6 & 591 & 100,0 \\
\hline
\end{tabular}

Legenda: (+) positivo; (-) negativo.

Tabela 2: Linhagens identificadas na cultura segundo os critérios de solicitação

\begin{tabular}{|c|c|c|c|c|c|c|c|c|}
\hline \multirow[t]{2}{*}{ Principais linhagens } & \multicolumn{2}{|c|}{ Critério 1} & \multicolumn{2}{|c|}{ Critério 4} & \multicolumn{2}{|c|}{ Demais critérios } & \multicolumn{2}{|c|}{ Total } \\
\hline & $\mathrm{n}^{\circ}$ & $\%$ & $\mathrm{n}^{\circ}$ & $\%$ & $\mathrm{n}^{\circ}$ & $\%$ & $\mathrm{n}^{\circ}$ & $\%$ \\
\hline MRSA & 38 & 46,9 & 5 & 10,9 & 16 & 61,5 & 59 & 38,3 \\
\hline Klebsiella pneumoniae & 17 & 21,0 & 23 & 50,0 & 8 & 30,8 & 48 & 31,2 \\
\hline Acinetobacter baumannii & 18 & 22,2 & 13 & 28,3 & 8 & 30,8 & 39 & 25,3 \\
\hline VRE & 14 & 17,3 & 11 & 23,9 & 3 & 11,5 & 28 & 18,2 \\
\hline Pseudomonas aeruginosa & 2 & 2,5 & 5 & 10,9 & 2 & 7,7 & 9 & 5,8 \\
\hline Entecrobacter cloacae & 3 & 3,7 & 5 & 10,9 & 1 & 3,8 & 9 & 5,8 \\
\hline Stenotrophomonas maltophilia & 2 & 2,5 & 3 & 6,5 & & 0,0 & 5 & 3,2 \\
\hline BGN não fermentador & 3 & 3,7 & & 0,0 & & 0,0 & 3 & 1,9 \\
\hline Escherichia coli & 2 & 2,5 & 1 & 2,2 & & 0,0 & 3 & 1,9 \\
\hline Serratia sp. & 1 & 1,2 & & 0,0 & 1 & 3,8 & 2 & 1,3 \\
\hline Proteus mirabilis & 2 & 2,5 & & 0,0 & & 0,0 & 2 & 1,3 \\
\hline Pantoae agglomerans & 1 & 1,2 & 1 & 2,2 & & 0,0 & 2 & 1,3 \\
\hline Providencia stuart & & 0,0 & 1 & 2,2 & & 0,0 & 1 & 0,6 \\
\hline Citrobacter freundii & & 0,0 & 1 & 2,2 & & 0,0 & 1 & 0,6 \\
\hline Total amostras positivas & 81 & & 46 & & 26 & & 150 & \\
\hline
\end{tabular}

Legenda: MRSA - Staphylococcus aureus resistente à meticilina; VRE -Enterococcus resistente à Vancomicina; BGN Bactérias Gram-Negativas

Tabela 3: Resultado das culturas, segundo critérios e faixas etárias

\begin{tabular}{lccccccccccc}
\hline Faixa etária (anos) & \multicolumn{2}{c}{ Critério 1} & \multicolumn{2}{c}{ Critério 4} & \multicolumn{2}{c}{ Demais critérios } & Sem informação & Total \\
\hline & $(+)$ & $(-)$ & $(+)$ & $(-)$ & $(+)$ & $(-)$ & $(+)$ & $(-)$ & $(+)$ & $(-)$ \\
\hline 10 & 0 & 10 & 0 & 0 & 1 & 1 & 0 & 4 & 1 & 15 \\
\hline $10-19$ & 3 & 13 & 0 & 3 & 1 & 0 & 2 & 1 & 6 & 17 \\
\hline $20-39$ & 5 & 81 & 4 & 9 & 2 & 1 & 1 & 16 & 12 & 107 \\
$40-59$ & 32 & 93 & 20 & 16 & 3 & 1 & 5 & 19 & 60 & 129 \\
$60-79$ & 28 & 93 & 13 & 34 & 2 & 6 & 9 & 16 & 52 & 149 \\
$80-102$ & 13 & 17 & 5 & 2 & 0 & 2 & 1 & 3 & 19 & 24 \\
Total & 81 & 307 & 42 & 64 & 9 & 11 & 18 & 59 & 150 & 441 \\
\hline
\end{tabular}

Legenda: (+) positivo; (-) negativo. 
Tabela 4: Média, mediana e desvio padrão da idade dos pacientes, segundo critérios e resultado da cultura

\begin{tabular}{lccccccc}
\hline Critérios & \multicolumn{3}{c}{ Resultado positivo } & \multicolumn{3}{c}{ Resultado negativo } & p valor \\
\hline & $M$ & Media. & DP & M & Media. & DP & 20,4 \\
Critério 1 & 60,4 & 60 & 17,6 & 49,9 & 51 & $<0,0001$ \\
Critério 4 & 58,1 & 58 & 19,4 & 55,8 & 61 & 19,8 & 0,5567 \\
Demais critérios & 55,0 & 56 & 22,1 & 60,0 & 65 & 22,2 & 0,3241 \\
Total & 57,9 & 59 & 19,3 & 50,8 & 54 & 20,7 & 0,0002 \\
\hline
\end{tabular}

Legenda: M - média; Media. - Mediana; DP - Desvio Padrão

Tabela 5: Resultados de cultura segundo sexo e critérios

\begin{tabular}{|c|c|c|c|c|}
\hline Critérios & Masc. & Fem. & DP & IC \\
\hline \multicolumn{5}{|l|}{ Critério 1} \\
\hline Positivo & 43 & 38 & & $0,8037-1,2750$ \\
\hline Negativo & 161 & 146 & & \\
\hline \multicolumn{5}{|l|}{ Critério 4} \\
\hline Positivo & 27 & 19 & 1,3358 & \\
\hline Negativo & 29 & 37 & & $0,9276-1,9238$ \\
\hline \multicolumn{5}{|c|}{ Demais critérios } \\
\hline Positivo & 5 & 4 & 1,2222 & \\
\hline Negativo & 5 & 6 & & $0,5110-2,9235$ \\
\hline \multicolumn{5}{|c|}{ Sem definição critério } \\
\hline Positivo & 10 & 8 & 0,9641 & \\
\hline Negativo & 34 & 25 & & $0,6040-1,5387$ \\
\hline \multicolumn{5}{|l|}{ Total } \\
\hline Positivo & 85 & 69 & 1,0589 & \\
\hline Negativo & 233 & 214 & & $0,8953-1,2523$ \\
\hline
\end{tabular}

Legenda: Masc. - Masculino; Fem. - Feminino; DP - Desvio Padrão; IC - Intervalo de confiança.

\section{DISCUSSÃO}

Neste estudo, o Critério 1- Transferidos de outra instituição com permanência maior que 96 horas foi o mais frequente. Moraes et al. (2013) em estudo realizado num grande hospital do município de São Paulo verificaram que a transferência de outro hospital e a internação na UTI foram fatores preditivos para cultura positiva para MRSA, Acinetobacter Baumannii e Klebsiella pneumoniae resistentes aos carbapenêmicos. Em investigação sobre os fatores de risco para IRAS, em um centro de terapia intensiva de um hospital de ensino, verificou-se que pacientes com internação prévia no CTI apresentaram risco de 1,93 (IC: $1,48-2,49$ ) para 0 desenvolvimento de infecções quando comparados aos procedentes da comunidade (OLIVEIRA; KOVNER; SILVA, 2010). Em estudo realizado no Hospital de Câncer de Barretos, observou-se que os critérios clínicos adotados para a instituição das precauções de contato dos pacientes, provenientes de outras instituições de saúde, apresentaram sensibilidade de $90,0 \%$, e especificidade de $6,5 \%$ (CATANEO et al., 2011).

Estudos diversos demonstraram os vários fatores de risco relacionados à aquisição de infecções por microrganismos resistentes, considerando o tempo de internação, realização de procedimentos invasivos (cirurgias, transplantes, cateter venoso, sonda vesical, ventilação mecânica), uso prévio de antimicrobianos (vancomicina, cefalosporinas de terceira e quarta gerações, carbapenêmicos, metronidazol e clindamicina), gravidade do quadro clínico, proximidade com pacientes colonizados ou infectados (ALTOPARLAK et al., 2011; BATISTÃO et al., 2012; HAYAKAWA et al., 2012; SANTAYANA et al., 2012).

Em estudo realizado na Austrália (MARSHALL; RICHARDS; MCBRYDE, 2013), houve $60 \%$ de redução na aquisição de MRSA após a identificação rápida de pacientes colonizados e instituição de medidas de precaução de contato. Em estudos onde se introduziu a realização da cultura de vigilância rotineiramente, verificou-se importante redução na incidência de VRE e nos custos com a assistência (MONTECALVO et al., 2001; MUTO et al., 2002; SHADEL et al., 2006). Segundo Shadel et al. (2006), o custo com a internação de um paciente que desenvolve IRAS é muito superior aos gastos que a instituição teria com a implementação da cultura de vigilância com tudo que inclui esta prática (tempo do profissional, paramentações, kits para swab, meio de 
cultura, entre outros)

A incidência de infecção por MRSA reduziu significativamente quando da implantação da cultura de vigilância e estabelecimento simultâneo das barreiras de precaução preventivas em um hospital de Boston (HUANG et al., 2006). A vigilância ativa por meio de coleta de swab nasal e de orofaringe têm contribuído para a redução nas taxas de infecção (SANTOS; FONSECA; GONTIJO FILHO, 1996).

Em estudo realizado na Polônia, utilizando o método de vigilância ativa de IRAS em pacientes adultos que passaram mais de 48 horas em uma enfermaria geral de UTI entre 2007 e 2016, os microrganismos mais identificados foram: Acinetobacter baumannii (25\%), seguido por Staphylococcus coagulase-negativa (15\%), Escherichia coli (9\%) (KOŁPA et al., 2018). Em relação aos resultados do swab anal, os microrganismos mais frequentemente identificados num hospital de Barretos (CATANEO et al., 2011) foram Staphylococcus aureus $(32,8 \%)$, Escherichia coli $(4,95)$ e Klebsiella spp.(6,6\%).

A cultura de vigilância ativa pode ser utilizada para direcionar a padronização dos antimicrobianos da instituição, uma vez que há um risco maior dos pacientes já colonizados desenvolverem alguma das IRAS com o microrganismo já existente na sua pele.

As medidas de precaução tomadas com o paciente com cultura positiva devem prolongar enquanto existir o estado de portador, o que geralmente ocorre durante toda a internação (RODRÍGUEZ-BAÑO et al., 2008).

O sexo dos pacientes não foi um fator relevante para ser submetido ou não à cultura de vigilância ativa nesse estudo. Em estudo realizado por Kołpa et al. (2018) o sexo masculino foi predominante entre os pacientes com resultado de cultura positiva. A média de idade dos pacientes com resultado de cultura positiva foi de 57,9 anos, semelhante à de alguns estudos realizados com adultos (CATANEO et al., 2011; KOŁPA et al., 2018).

\section{CONCLUSÃO}

A implementação da precaução de contato de forma preliminar atua de forma complementar, já que a proximidade entre pacientes e profissionais de saúde durante a assistência destes é um relevante fator de risco para a continuidade da transmissão de microrganismos.

A partir dos resultados, criou-se um banco de dados das culturas de vigilância que possibilita a identificação do paciente com cultura positiva em internações anteriores. O SCIH e a equipe assistencial podem identificar aqueles já colonizados/infectados e instaurar as medidas de controle logo na admissão dos mesmos (uso de avental e luvas). O paciente já sabidamente colonizado com bactérias multirresistentes será submetido à nova coleta de cultura de vigilância se preencher um dos critérios pré-definidos pelo $\mathrm{SCIH}$. Caso o paciente não preencha os critérios, mas apresenta resultado positivo há menos de três meses, para qualquer bactéria exceto VRE, será mantido em precaução de contato. Se foi positivo para VRE, esse prazo de manutenção da precaução de contato passa a ser 06 meses.

Essas condutas reduzem a possibilidade de transmissão cruzada e realização de culturas desnecessárias.

Os resultados encontrados permitiram conhecer o perfil microbiológico e epidemiológico dos pacientes admitidos no hospital e contribuiu para maior adesão às medidas de precauções de contato. Além disso, demonstraram a importância da permanência do protocolo da cultura de vigilância ativa na instituição, com vistas à prevenção e controle das IRAS.

\section{REFERÊNCIAS}

AGÊNCIA NACIONAL DE VIGILÂNCIA SANITÁRIA (ANVISA). Investigação e controle de bactérias multirresistentes. Brasília: Anvisa, 2007.

ALTOPARLAK, U. et al. Incidence and risk factors of vancomycinresistant enterococcus colonization in burn unit patients. Burns: Journal of the International Society for Burn Injuries, v. 37, n. 1 , p. $49-53$, feb. 2011.

ÁLVAREZ LERMA, F. et al. Optimization of pre-emptive isolations in a polyvalent ICU through implementation of an intervention strategy. Medicina Intensiva, v. 39, n. 9, p. 543-551, dec. 2015.

BATISTÃO, D. W. F. et al. Risk factors for vancomycin-resistant enterococci colonisation in critically ill patients. Memórias do Instituto Oswaldo Cruz, v. 107, n. 1, p. 57-63, 2012.

BRASIL. Ministério da Saúde. Portaria no 2.616, de 12 de maio de 1998. Expede na forma dos anexos I, II, III, IV, V diretrizes e normas para a prevenção e o controle das infecções hospitalares tais como: herpes simples, toxoplasmose, rubeola, citomegalovirose, sífilis, AIDS. Diário Oficial da União, Brasília, 13 maio 1998. Seção 1, p. 133.

CATANEO, $C$ et al. Evaluation of the sensitivity and specificity of criteria for isolation of patients admitted to a specialized cancer hospital. Revista Latino-Americana de Enfermagem, v. 19, n. 5, p. 1072-1079, 2011.

CURSINO, M. A. et al. Performance of surveillance cultures at different body sites to identify asymptomatic staphylococcus aureus carriers. Diagnostic Microbiology and Infectious Disease, v. 74, n. 4, p. 343-348, dec. 2012.

DALBEN, M. F. et al. Swab cultures across three different body sites among carriers of carbapenem-resistant $\mathrm{p}$. aeruginosa and acinetobacter species: a poor surveillance strategy. The Journal of Hospital Infection, v. 74, n. 4, p. 395-396, apr. 2010.

FIGUEIREDO, D. A. F. Fatores de risco associados à infecção hospitalar em uma unidade de terapia intensiva. 2012. 101 f. Dissertação (Mestrado em Modelos de Decisão e Saúde) - 
Universidade Federal da Paraíba, João Pessoa, 2012.

FURUNO, J. P. et al. Identifying groups at high risk for carriage of antibiotic-resistant bacteria. Archives of Internal Medicine, v. 166, n. 5, p. 580-585, mar. 2006.

HAYAKAWA, K. et al. Comparison of the clinical characteristics and outcomes associated with vancomycin-resistant Enterococcus Faecalis and Vancomycin-Resistant E. Faecium Bacteremia. Antimicrobial Agents and Chemotherapy, v. 56, n. 5, p. 2452-2458, may. 2012.

HUANG, S. S. et al. Impact of routine intensive care unit surveillance cultures and resultant barrier precautions on hospital-wide methicillin-resistant staphylococcus aureus bacteremia. Clinical Infectious Diseases, v. 43, n. 8, p. 971978, oct. 2006.

KOŁPA, M. et al. Incidence, microbiological profile and risk factors of healthcare-associated infections in intensive care units: a 10 year observation in a provincial hospital in Southern Poland. International Journal of Environmental Research and Public Health, v. 15, n. 1, jan. 2018.

LACHHAB, Z. et al. Bacteraemia in intensive care unit: clinical, bacteriological, and prognostic prospective study. Canadian Journal of Infectious Diseases and Medical Microbiology, v. 2017, p. 1-9, mar. 2017.

MARSHALL, C.; RICHARDS, M.; MCBRYDE, E. Do active surveillance and contact precautions reduce MRSA acquisition? A prospective interrupted time series. PloS One, v. 8, n. 3, p. e58112, mar. 2013.

MARTINS, A. F. et al. Controle e monitoramento de microrganismos multirresistentes. Porto Alegre: Procempa, 2014. Disponível em: http://lproweb.procempa.com.br/pmpa/prefpoa/cgvs/ usu_doc/controle_e_monitoramento_de_microrganismos_ multirresistentes.pdf. Acesso em: 14 jan. 2019.

MONTECALVO, M. A. et al. Costs and savings associated with infection control measures that reduced transmission of vancomycin-resistant enterococci in an endemic setting. Infection Control and Hospital Epidemiology, v. 22, n. 7, p. 437-442, jul. 2001.

MORAES, G. M. et al. Infecção ou colonização por microorganismos resistentes: identificação de preditores. Acta Paulista de Enfermagem, v. 26, n. 2, p. 185-191, 2013.

MORGAN, D. J. et al. Improving efficiency in active surveillance for methicillin-resistant staphylococcus aureus or vancomycinresistant enterococcus at hospital admission. Infection Control and Hospital Epidemiology, v. 31, n. 12, p. 1230-1235, dec. 2010.

MUTO, C. A. et al. Cost-effectiveness of perirectal surveillance cultures for controlling vancomycin-resistant enterococcus. Infection Control and Hospital Epidemiology, v. 23, n. 8, p. 429-435, aug. 2002.
NOGUEIRA, P. S. F. et al. Perfil da infecção hospitalar em um hospital universitário. Revista de Enfermagem da UERJ, v. 17, n. 1 , p. 96-101, mar. 2009.

OLIVEIRA, A. C.; KOVNER, C. T.; SILVA, R. S. Infecção hospitalar em unidade de tratamento intensivo de um hospital universitário brasileiro. Revista Latino-Americana de Enfermagem, v. 18, n. 2, p. 97-104, 2010.

PAIM, R. S. P.; LORENZINI, E. Estratégias de prevenção da resistência bacteriana. Revista Cuidarte, v. 5, n. 2, p. 757-764, 2014.

REVIEW ON ANTIMICROBIAL RESISTANCE. Antimicrobial Resistance: Tackling a Crisis for the Health and Wealth of Nations. 2014. Disponível em: https://amr-review.org/sites/default/files/ AMR\%20Review\%20Paper\%20-\%20Tackling\%20a\%20crisis\%20 for\%20the $\% 20$ health\%20and\%20wealth\%20of\%20nations_1. pdf. Acesso em 4 fev. 2019.

RODRÍGUEZ-BAÑO, J. et al. Community infections caused by extended-spectrum beta-lactamase-producing escherichia coli. Archives of Internal Medicine, v. 168, n. 17, p. 1897-1902, 2008.

SANTAYANA, E. M. et al. Risk factors and outcomes associated with vancomycin-resistant enterococcus infections with reduced susceptibilities to linezolid. Diagnostic Microbiology and Infectious Disease, v. 74, n. 1, p. 39-42, sep. 2012

SANTOS, K. R. N.; FONSECA, L. S.; GONTIJO FILHO, P. P. Emergence of high-level mupirocin resistance in methicillinresistant staphylococcus aureus isolated from Brazilian University Hospitals. Infection Control and Hospital Epidemiology, v. 17 , n. 12 , p. 813-816, dec. 1996

SHADEL, B. N. et al. Surveillance for vancomycin-resistant enterococci: type, rates, costs, and implications. Infection Control and Hospital Epidemiology, v. 27, n. 10, p. 10681075, oct. 2006. 